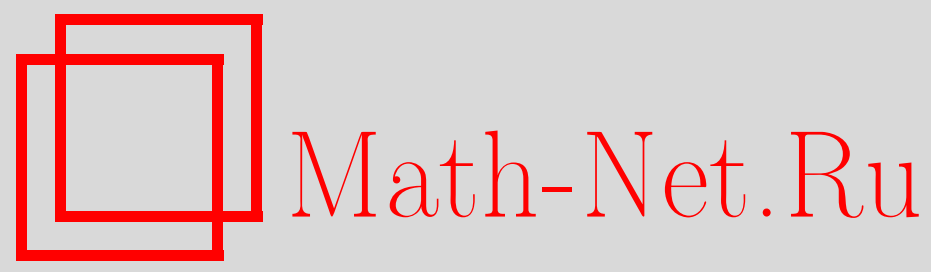

Б.Ф. Мельников, Об $\omega$-языках специальных биллиардов, Дискрет. матем., 2002, том 14, выпуск 3, 95-108

DOI: https://doi.org/10.4213/dm257

Использование Общероссийского математического портала Math-Net.Ru подразумевает, что вы прочитали и согласны с пользовательским соглашением http://www.mathnet.ru/rus/agreement

Параметры загрузки:

IP : 54.172 .240 .79

26 апреля 2023 г., 11:56:59 
удк 519.6

\title{
Об $\omega$-языках специальных биллиардов
}

\author{
( 2002 г. . Б. Ф. Мельников
}

\begin{abstract}
В статье рассматриваются недетерминированные инициальные конечные автоматы без финальных состояний и $\omega$-языки, определяемые такими автоматами. Для таких $\omega$-языков рассматриваются так называемые языки обструкций. Определяются и подробно рассматриваются биллиардные $\omega$-языки, задаваемые специальным образом для каждого $n \geqslant 3$ над алфавитом из $n$ букв. Каждое $\omega$-слово такого $\omega$-языка может быть получено с помощью бесконечного числа правильных отражений точки от стенок биллиарда, имеющего форму правильного $n$-угольника. Для таких $\omega$-языков рассматриваются языки обструкций и показывается, что ни при каком $n$ такой язык обструкций не является регулярным.

Работа выполнена при поддержке Российского фонда фундаментальных исследований, проекты 99-01-00907 и 00-15-99253.
\end{abstract}

\section{1. Введение}

В статье рассматриваются слова (конечные слова) и $\omega$-слова (бесконечные слова) над заданным конечным алфавитом $\Sigma$. Каждое $\omega$-слово можно рассматривать, например, как функцию вида

$$
\sigma: \mathbf{N} \rightarrow \Sigma .
$$

Например, можно рассмотреть простейшее $\omega$-слово $a^{\omega}$, каждая буква которого есть $a$, то есть функщия $\sigma$ здесь такова, что $\sigma(i)=a$ для всех $i \in \mathbf{N}$. Более подробные определения и примеры имеются в [1-4].

Мы также будем рассматривать недетерминированные конечные автоматы РабинаСкотта (Медведева), определенные согласно [5]; для таких автоматов мы будем пользоваться сокращением НРС-автоматы.

Данную работу можно считать продолжением [6]. В [6] были рассмотрены языки (множества) обструкций (по поводу терминологии см. $[3,4]$ ) для $2 \omega$-языков, определяемых НРС-автоматами, не имеющими ни стартовых (инищиальных), ни финальных состояний. Заметим, что похожие на языки обструкций объекты рассматриваются и в $[7,8,9]$.

В отличие от [6] мы будем рассматривать НРС-автоматы, имеющие стартовые состояния (для них будем использовать сокращение $\omega$-КА) и, следовательно, не $2 \omega$-языки, а $\omega$-языки. Для последних возможны практически те же самые задачи, которые были рассмотрены в [6] для $2 \omega$-языков, мы кратко рассмотрим эти задачи в следующем разделе.

Главная проблема, рассматривающаяся в данной статье, это исследование не $\omega$-языков вообще, а специальных $\omega$-языков. А именно, для каждого $n \geqslant 3$ будем рассматривать $\omega$-язык, каждое $\omega$-слово которого может быть определено с помощью биллиарда, являющегося правильным $n$-угольником. Оно определяется как некоторая бесконечная 
последовательность правильных отражений точки от стен данного биллиарда, точнее, бесконечная последовательность меток этих стен. Для подобных $\omega$-языков в статье обычным образом определяются языки обструкций и показывается, что ни при каком $n \geqslant 3$ такой язык обструкций не является регулярным.

\section{2. Основные определения и предварительные замечания}

Как уже было сказано выше, в [6] были определены языки обструкций для $2 \omega$-языков. Доказано, что если $2 \omega$-язык определяется некоторым $2 \omega$-автоматом, то язык обструкций этого $2 \omega$-языка регулярен. И наоборот, для каждого регулярного языка $L$, не содержащего никакого своего слова в качестве подслова некоторого другого слова этого языка, существует некоторый $2 \omega$-КА, чье множество обструкций совпадает с языком $L$. Дополнительное требование, накладываемое здесь на регулярный язык, формулируется очень громоздко. Заметим однако, что при некоторых обстоятельствах (несколько другом определении множества обструкщий) оно может быть опущено, подробнее см. [6]. Поэтому не является ошибкой и следующая более простая фраза: для каждого регулярного языка $L$ существует некоторый $2 \omega$-КА, чье множество обструкций совпадает с $L$.

В этом разделе кратко рассматриваются задачи, известные по [1-4] и [10-14] и, как уже было отмечено, являющиеся формулировками практически тех же самых задач для $\omega$-языков и $\omega$-КА. В связи с разными обозначениями, встречающимися в процитированных работах, приведем определения с самого начала, то есть рассмотрим некоторый $\omega$-КА (НРС-автомат, имеющий стартовые состояния и не имеющий финальных) и определим обычным образом $\omega$-язык и язык обструкций этого автомата. Мы будем использовать обозначения, близкие к [6].

Отметим еще, что многие из утверждений, приводимых далее в данном разделе, могут быть доказаны не только на основе вышеуказанных работ, но и на основе [6]. Будем формулировать эти утверждения как теоремы; возможные доказательства см. в [1-4] и [10-14].

\section{1. $\omega$-автоматы}

Определение 1. Под $\omega$-автоматом понимается четверка $K_{\omega}=(Q, \Sigma, \delta, S)$, где $\Sigma-$ рассматриваемый алфавит, $Q-$ множество состояний, $S \subseteq Q-$ множество стартовых состояний, $\delta$ - функщия вида,

$$
\delta: Q \times \Sigma \rightarrow \mathscr{P}(Q),
$$

где здесь и далее $\mathscr{P}(A)$ - множество всех подмножеств множества $A$.

Определение 2. Будем считать, что $\omega$-слово, определяемое некоторой функцией $\sigma: \mathbf{N} \rightarrow \Sigma$, принадлежит языку некоторого $\omega$-КА $(Q, \Sigma, \delta, S)$ тогда и только тогда, когда существует некоторая функция $q: \mathbf{N} \cup\{0\} \rightarrow Q$ (значения этой функция - бесконечная последовательность состояний заданного автомата), для которой выполнены следующие условия: $q(0) \in S$ и для любого $i \in \mathbf{N}$ выполнено условие

$$
\delta(q(i-1), \sigma(i)) \ni q(i) .
$$

Ниже на рисунках будем приводить графы переходов $\omega$-КА согласно [5] однако без выходных стрелок, заметим, что такое представление немного отличается от принятого как в отечественной литературе, так, например, и в [15]. 


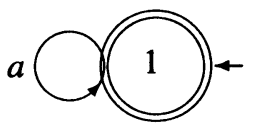

Pис. 1.

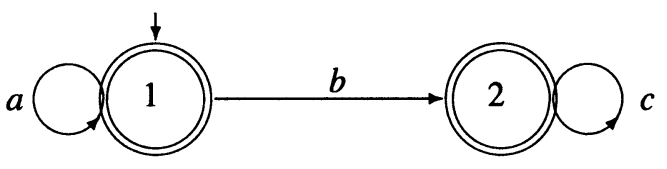

Рис. 2.

Простейший $\omega$-КА (определяющий $\omega$-язык $\left\{a^{\omega}\right\}$, содержащий только одно $\omega$-слово) приведен на рис. 1. Число 1 использовано для обозначения названия состояния (оно стартовое, поэтому помечено входной стрелкой), $a$ - метка дуги. А автомат на рис. 2 определяет $\omega$-язык, содержащий $a^{\omega}$, а также все $\omega$-слова вида

$$
a a a \ldots a a a b c c c \ldots c c c \ldots
$$

Мы можем записать этот $\omega$-язык как

$$
a^{\omega}+a^{*} b c^{\omega}
$$

Не будем строго определять подобный способ обозначений, он аналогичен обычным регулярным выражениям. Некоторое обобщение см. в [6].

Определение 3. Будем называть функщию переходов $\delta$ детерминированной, если для любой возможной пары ее аргументов значение функции состоит не более, чем из одного состояния.

Некоторый $\omega$-КА будем также называть детерминированным, если его множество стартовых состояний состоит не более, чем из одного элемента, а функция переходов $\delta$ является детерминированной.

Заметим, что оба автомата, рассмотренные в примерах этого раздела, являются детерминированными.

\section{2. Обструкции}

Определение 4. Пусть $\mathscr{A}$ - некоторый $\omega$-язык. Будем называть слово $u \in \Sigma^{*}$ обобщенной обструкцией языка $\mathscr{A}$, если не существует никакого $\omega$-слова $\alpha \in \mathscr{A}$, содержащего $u$ в качестве подслова, то есть если

$$
(\forall \alpha \in \mathscr{A})\left(\forall v \in \Sigma^{*}, \gamma \in \Sigma^{\omega}\right)(\alpha \neq v u \gamma) .
$$


Слово $u \in \Sigma^{*}$, являющееся обобщенной обструкцией языка $\mathscr{A}$, будем называть (собственно) обструкцией, если не существует другого слова $v \in \Sigma^{*}$, являющегося обобщенной обструкщией языка $\mathscr{A}$, такого, что $v$ - подслово слова $u$, то есть если

$$
\left(\forall w_{1}, w_{2} \in \Sigma^{*}\right)\left(u \neq w_{1} v w_{2}\right)
$$

Множество всех (собственно) обструкщий заданного $\omega$-языка $\mathscr{A}$ будем обозначать $\mathcal{O}(\mathscr{A})$.

Заметим, что практически всегда мы можем предполагать, не ограничивая общности, что каждая (собственно) обструкция содержит не менее 2 букв, в противном случае мы можем просто исключить букву-обструкцию из рассматриваемого алфавита $\Sigma$.

Рассмотрим примеры. Мы будем рассматривать только такие $\omega$-языки, которые могут быть определены с помощью $\omega$-КА. Очевидно, существуют и другие $\omega$-языки (просто потому, что множество $\omega$-КА счетно, а множество $\omega$-языков над алфавитом, содержащим более 1 буквы, более, чем счетно). Подробнее см. [13].

Для $\omega$-языка $\mathscr{A}=\left\{a^{\omega}\right\}$ над алфавитом $\{a\}$ множество $\mathcal{O}(\mathscr{A})$ пустое, поскольку этот язык содержит все $\omega$-слова над $\Sigma$. Вообще, если этот же $\omega$-язык $\mathscr{A}$ рассматривать над алфавитом $\Sigma$, содержащим более одной буквы, то $\mathcal{O}(\mathscr{A})=\Sigma \backslash\{a\}$ (например, для $\Sigma=\{a, b\}$ получаем, что $\mathcal{O}(\mathscr{A})=\{b\})$. Мы можем также доказать, что для $\omega$-языка $\mathscr{B}$, определяемого автоматом на рис. 2 , язык обструкций $\mathcal{O}(\mathscr{B})$ есть $\{a c, b a, b b, c a, c b\}$. Этот факт может быть получен с помощью теорем, доказанных в [6]. Однако рассматриваемый $\omega$-язык довольно прост, и мы можем обойтись без этих теорем. Действительно, мы можем рассматривать только слова, содержащие 2 буквы или более. Среди 2-буквенных слов (их 9) 5 слов, это вышеупомянутые

$$
B=\{a c, b a, b b, c a, c b\},
$$

являются обструкциями согласно определению 4 , а остальные 4 слова не удовлетворяют этому определению. Среди слов, состоящих из 3 или более букв, не содержат слов из языка $B$ только те, которые могут быть определены регулярным выражением

$$
a^{*}+a^{*} b c^{*}+c^{*}
$$

(этот факт можно получить на основе рис. 2). Но, по определению рассматриваемого $\omega$-языка, все слова из последнего множества не являются обструкциями.

Во всех рассмотренных выше примерах языки $\mathcal{O}(\mathscr{A})$ конечные, однако существуют примеры $\omega$-автоматов, для которых множества $\mathcal{O}(\mathscr{A})$ бесконечны. Автору не известны простые примеры, однако они могут быть получены согласно [6], точнее с помощью обобщения теорем, доказанных в [6], на случай $\omega$-языков, либо согласно следующих двух теорем (см. [3, 4]).

Теорема 1. Пусть некоторый $\omega$-язык $\mathscr{A}$ может быть определен с помощью $\omega-K A$. Тогда язык $\mathcal{O}(\mathscr{A})$ регулярен.

Теорема 2. Пусть язык L регулярен. Тогда язык

$$
M=L \backslash\left\{u \in L \mid\left(\exists v \in L, w_{1}, w_{2} \in \Sigma^{*}\right)\left(w_{1} w_{2} \neq e, u=w_{1} v w_{2}\right)\right\}
$$

также регулярен и существует $\omega$-язык $\mathscr{A}$, который можно определить с помощью некоторсго $\omega-K A$, для которого $\mathcal{O}(\mathscr{A})=M$. 


\section{3. Автоматы со счетным числом состояний}

Далее мы часто будем рассматривать специальное обобщение введенного множества $\omega$-автоматов, а именно, случай, когда множество состояний $Q$ может быть бесконечным (счетным), подробнее см. $[10,11]$. Для этих автоматов $\omega$-языки и языки обструкций определяются обычным образом. Комбинируя определения из [1] и [16], мы можем определить $\omega$-языки, задаваемые $\omega$-автоматами, двумя другими способами, либо как 1-допущение с множеством отмеченных состояний, являющимся полным множеством $\mathscr{P}(Q)$, либо как $\lim (\mathscr{L})$, где обозначение $\lim$ употреблено согласно [16], а $\mathscr{L}$ - язык обычного НРС-автомата, соответствующего заданному $\omega$-КА, со множеством финальных состояний, совпадающим с $Q$. Отметим еще, что и для $\lim (L)$ также имеются альтернативные обозначения, обозначение $\bar{L}$, употребляемое в [3] и $L^{\delta}$, употребляемое в [4].

Заметим, что даже такие автоматы не могут определять любой $\omega$-язык, поскольку множество $\omega$-языков более чем счетно. Также обычным образом определим выходные $\omega$-языки каждого состояния, а именно, для $\omega$-автомата $(Q, \Sigma, \delta, S)$ и состояния $q \in Q$ выходной $\omega$-язык определяется $\omega$-автоматом $(Q, \Sigma, \delta,\{q\})$.

Далее будем рассматривать детерминированные $\omega$-автоматы. Если 2 (или более) состояния такого детерминированного $\omega$-КА имеют один и тот же выходной $\omega$-язык, то их можно назвать эквивалентными и объединить в одно (аналогично тому, как это делается в теории обычных конечных автоматов, см., например, [5], [17]). Верна следующая теорема.

Теорема 3. Пусть $\mathcal{K}_{\omega}=(Q, \Sigma, \delta, S)$ - детерминированный $\omega-K A$ с бесконечным множеством состояний $Q$. Тогда его язык может быть определен некоторым $\omega-К А$ с конечным множеством состояний тогда и только тогда, когда существует разбиение множества $Q$ на конечное число подмножеств эквивалентных состояний.

\section{3. языки обструкций}

Для каждого $n \geqslant 3$ рассмотрим биллиард в форме правильного $n$-угольника. Пусть его стороны помечены числами $0,1, \ldots, n-1$ (эти метки можно рассматривать как цифры $n$-ичной системы счисления и, следовательно, как буквы некоторого алфавита).

Далее, рассмотрим некоторую бесконечную траекторию точки в таком биллиарде, запущенной с обычным законом отражения (без затухания). Заметим, что мы можем не рассматривать траектории, заканчивающиеся в каком-либо углу. Действительно, таких заканчивающихся траекторий - счетное множество, поэтому существуют и бесконечные. Рассмотрим (бесконечную) последовательность меток сторон, где отразилась точка в процессе движения по этой траектории; такая последовательность меток является $\omega$-словом над алфавитом $\Sigma_{n}=\{0,1, \ldots, n-1\}$. Все возможные $\omega$-слова для заданного многоугольника образуют специальный $\omega$-язык (будем называть его $\omega$-языком заданного многоугольника). Иногда, когда важно число сторон $n$, будем говорить об $\omega$-языке правильного $n$-угольника. Будем называть язык обструкций последнего $\omega$-языка языком обструкщий многоугольника. 


\section{4. Фигуры отражений и специальные $\omega$-автоматы}

\section{1. Фигуры отражений}

Зафиксируем какие-нибудь две стороны заданного правильного многоугольника; пусть эти стороны помечены буквами (цифрами) $a$ и $b$. Для этих сторон рассмотрим некоторый прямоугольник, стороны которого будем рассматривать как две координаты. Отметим заранее, что важно не путать механическую точку, движущуюся по заданному биллиарду, и точку введенного здесь прямоугольника. Первая из этих координат изменяется в границах $[0,1]$ и является координатой точки на стороне $a$, где произошло некоторое отражение точки в процессе движения по рассматриваемой траектории, а вторая координата изменяется в границах $[-\pi / 2, \pi / 2]$ и является углом отражения. Такие прямоугольники мы будем рассматривать для разных пар сторон. Поэтому будем считать для единообразия, что если рассматривать первую сторону (помеченную здесь $a$ ) изнутри правильного многоугольника, то первая координата равна 0 для самой правой ее точки, и 1 для самой левой. Аналогично желательно выбрать единое положительное направление для углов (вторых координат). Еще раз отметим, что в этом замечании приведены желательные, а не обязательные требования, поэтому более подробно и строго формулировать эти требования мы не будем (см. рисунки в последующем тексте).

Далее будем в этом прямоугольнике рассматривать только такие точки, для каждой из которых (то есть для пар, состоящих из координаты на стороне $a$ и угла отражения) следуюшая (после этого отражения) сторона есть $b$; как и ранее, мы не будем рассматривать попадания в углы, поэтому граничные точки стороны $b$ также считаем невозможными. Фигуру, образованную всеми подобными точками, будем называть фигурой для $(a-b)$-отражения.

Таким же способом мы можем определить фигуры для последовательности отражений, то есть для последовательности сторон, помеченных

$$
a_{1}, a_{2}, \ldots, a_{k} \in \Sigma \text {. }
$$

Такие фигуры, вообще говоря, могут быть пустыми в случае, если последовательность (1) включает в себя некоторую обструкцию как подслово (можно доказать, что это условие является не только достаточным, но и необходимым) или совпадать со всей внутренней областью прямоугольника при $k=1$. Будем называть фигуры для любой последовательности отражений (1) фигурами для $\left(a_{1}-a_{2}-\ldots-a_{k}\right)$-отражений.

Более того, мы можем строить подобные фигуры не только для двух координат первой стороны последовательности (1) (то есть не только для координат $a_{1}$ ), но и для координат, соответствующих $a_{i}$ при любом $i \in\{1, \ldots, k\}$. Будем называть такую фигуру фигурой отражений $a_{1} a_{2} \ldots a_{i} \ldots a_{k}$ (всегда подчеркнута ровно одна буква, именно та буква $a_{i}$, для координат которой строится фигура). То же самое немного подробнее отражено в следующем определении.

Определение 5. Рассмотрим последовательность отражений (1). Пусть такая последовательность возможна, при этом $i$-е отражение произошло от стороны, помеченной $a_{i}$, в точке с координатой $x \in[0,1]$ под углом $\alpha \in[-\pi / 2, \pi / 2]$. Тогда точка с координатами $(x, \alpha)$ входит в фигуру отражений $a_{1} a_{2} \ldots a_{i} \ldots a_{k}$. Больше ни одна точка в эту фигуру не входит.

Заметим, что уравнения для границ любой фигуры отражений можно выписать с помощью простых фактов элементарной математики. Сами границы в фигуру не входят, 

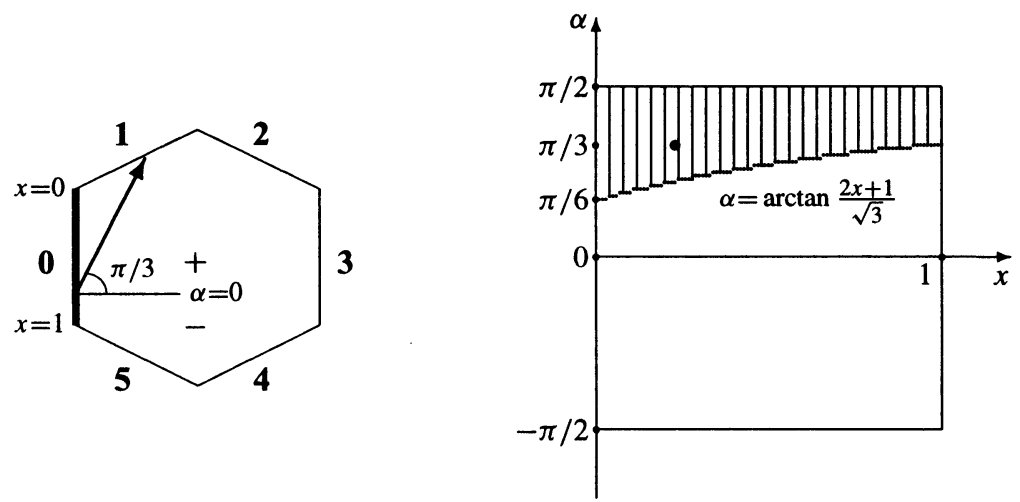

Рис. 3.

поскольку каждая из точек границы соответствует попаданию механической точки в один из углов заданного биллиарда. В том числе не входят те части границ, которые совпадают с границей прямоугольника $[0,1] \times[-\pi / 2, \pi / 2]$. Рассмотрим два примера. В обоих на левой половине рисунка будем приводить заданный биллиард, при этом сторону, соответствующую подчеркнутому отражению, выделять жирной линией и отмечать граничные значения первой координаты $x=0$ и $x=1$. Возможное движение механической точки будем изображать последовательностью векторов. На правой половине рисунка будут изображены прямоугольники $[0,1] \times[-\pi / 2, \pi / 2]$, где первая координата обозначена $x$, a вторая $\alpha$, а также фигуры отражений. Кроме того, на правой половине каждого рисунка будут приведены уравнения линий (вывод этих уравнений несложен и поэтому опущен), а также точка, соответствующая движению по последовательности векторов, отмеченной на левой половине рисунка.

В первом примере, пусть $n=6$; на рис. 3 приведена фигура отражений $\underline{0} 1$. На левой половине рисунка отмечено возможное значение угла отражения, $\pi / 3$. Во втором примере, для $n=4$, рассмотрим фигуру отражений $012 \underline{3} 0$; она приведена на рис. 4 . Заметим, что на этом рисунке мы не отметили угол $\alpha$ и его возможные значения на левой половине, а также границу прямоугольника $[0,1] \times[-\pi / 2, \pi / 2]$ на правой.

\section{2. Входные языки и выходные $\omega$-языки}

Рассмотрим некоторое слово $u$ (причем $u \neq e$ ), а также фигуру отражений этого слова с подчеркнутой последней буквой. Будем говорить, что каждая точка этой фигуры имеет входное слово $u$. Заметим, что входным словом для этой точки является и любой суффикс слова $u$. Образуемые этими входными словами языки аналогичны входным языкам состояний НРС-автомата, определенным в статье [18] и используемым в [6].

Другими словами, некоторое слово является входным словом для данной точки фигуры отражений с координатами $(x, \alpha)$, если последовательность букв этого слова можно рассматривать как последовательность отражений механической точки от сторон биллиарда, причем последнее отражение (то есть отражение от стороны, соответствующей последней букве) произошло в точке с координатой $x$ под углом $\alpha$. 

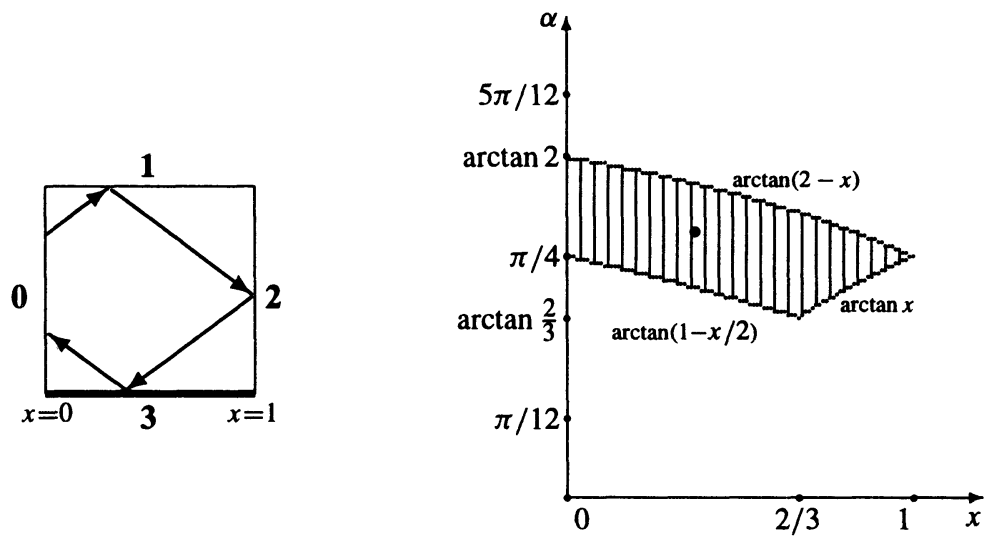

Pис. 4.

Аналогично, некоторые точки рассматриваемой фигуры отражений имеют выходные $\omega$-слова, причем каждая только одно.

Заметим, что мы уже фактически определили выходные $\omega$-слова в разделе 3 . Объединение всех выходных $\omega$-слов некоторой фигуры назовем выходным $\omega$-языком этой фигуры.

Предложение 1. Пусть для некоторых $u \in \Sigma^{*} u a \in \Sigma$ фигура отражений иа не пуста. Тогда эта фигура включает две различные точки, имеючие одну и ту же первую координату (и следовательно, включает весь отрезок с кончами в этих точках).

Приведем схему доказательства. Как было сказано выше, мы можем легко выписать уравнения границ фигуры, используя факты элементарной математики. Поскольку границы фигуры ей не принадлежат, для каждого значения первой координаты имеем ограничения, являющиеся строгими неравенствами. Последний факт доказывает утверждение.

Следующее утверждение можно рассматривать как инверсное к предыдущему. Однако, в отличие от [18], мы не можем рассматривать выходной язык некоторого состояния как инверсный (зеркальный) язык к входному языку того же состояния НРС-автомата, инверсного (зеркального) к данному, поскольку мы рассматриваем $\omega$-автоматы. Поэтому, кроме предложения 1 , мы формулируем и предложение 2.

Предложение 2. Пусть некоторая фигура отражений включает две различные точки, имеющие одну и ту же первую координату. Тогда существуют две различные точки этой фигуры с той же самой первой координатой, имеющие выходные $\omega$-слова; эти $\omega$-слова различны.

Автор располагает полным доказательством утверждения 2, однако оно довольно объемно и не является важным для материала данной статьи; поэтому оно опущено. Заметим лишь, что первая часть утверждения 2 очевидна. Далее в статъе используется частный случай этого утверждения, а именно, предложение 3 , которое и будет доказано ниже. 


\section{3. Соответствующие $\omega$-автоматы}

Зафиксируем некоторую стартовую букву алфавита (и, следовательно, некоторую стартовую сторону траектории) и рассмотрим детерминированный $\omega$-КА (с бесконечным множеством состояний), чьи состояния помечены всеми словами над алфавитом $\Sigma_{n}$, начинающимися с зафиксированной стартовой буквы. Ниже каждое состояние этого $\omega$-КА будет определять свою собственную фигуру отражений, а именно, ту фигуру, которая соответствует слову-метке этого состояния с подчеркнутой последней буквой. Будем называть эту фигуру входной фигурой (рассматриваемого состояния.) Заранее отметим, что можно не рассматривать состояния, чьи входные фигуры пусты (ниже так и будем делать).

Для таких автоматов стартовое состояние помечено зафиксированной стартовой буквой, а функция переходов $\delta$ определяется очевидным образом: для любых $u \in \Sigma^{*}$ и $a, b \in \Sigma$ мы имеем дугу из состояния, помеченного $u \underline{a}$ в состояние, помеченное иa $\underline{b}$ (если эти состояния имеются). По построению данного $\omega$-автомата, его $\omega$-язык совпадает с $\omega$-языком правильного $n$-угольника (поскольку, как уже было отмечено, мы не рассматриваем состояния, чьи входные фигуры пусты).

К сожалению, мы по понятным причинам не можем рассмотреть примеров таких $\omega$-автоматов.

Мы можем также определить и входные языки каждого состояния таких $\omega$-автоматов. Однако

- мы можем определить их обычным образом, то есть так, как это делается для обычных НРС-автоматов;

- каждый из таких входных языков включает единственное слово, уже рассмотренное выше, причем, здесь нет необходимости рассматривать все суффиксы этого слова, как мы делали выше, поскольку мы строим детерминированный $\omega$-автомат с единственным стартовым состоянием.

поэтому мы не будем приводить определения.

Как уже было сказано выше, выходные $\omega$-языки состояний таких $\omega$-КА не могут быть определены так же, как это делалось для обычных НРС-автоматов. Подробнее эти $\omega$-языки будут рассмотрены в оставшейся части статьи.

\section{5. Бесконечное множество различных входных фигур}

Зафиксируем некоторое $n \geqslant 3$, положим $m=n-1$ и рассмотрим $\omega$-слово

$$
\gamma_{n}=(012 \ldots m)^{\omega}=012 \ldots m 012 \ldots m \ldots
$$

Будем рассматривать префиксы $\gamma_{n}$ длины $k \geqslant 0$ и обозначать их $u_{n k}$. Остаток $\omega$-слова $\gamma_{n}$ без $u_{n k}$ (это снова $\omega$-слово) будем обозначать $\gamma_{n k}$.

Предложение 3. Две разные точки, которые можно рассматривать как точки фигуры отражений, соответствующей слову $u_{n k} c$ подчеркнутой последней буквой, имеющие одну и ту же первую координату, не могут иметь одно и то же выходное $\omega$-слово $\gamma_{n k}$.

Доказательство. Без ограничения общности мы можем предполагать, что $k=0$. Далее будем доказывать утверждение от противного, то есть предположим, что наше утверждение неверно и существуют две различные точки с одной и той же первой координатой (пусть $x_{0}$ ), имеющие одно и то же выходное $\omega$-слово, равное $\gamma_{n k}$. 


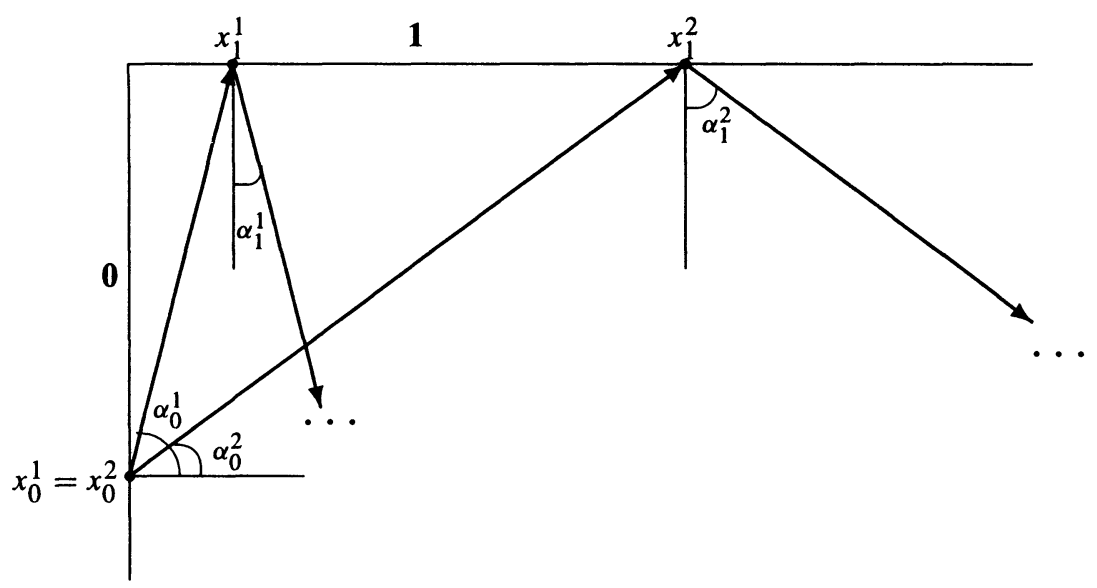

Рис. 5.

Пусть вторые координаты этих точек суть $\alpha_{0}^{1}$ и $\alpha_{0}^{2}$. Мы используем практически те же самые обозначения, которые употреблялись на рисунках 3 и 4 . Пусть также $\alpha_{0}^{1}>\alpha_{0}^{2}$. Теперь обозначим пары координат соответствующих траекторий как $\left(x_{i}^{j}, \alpha_{i}^{j}\right)$, где $j-$ номер точки (или траектории, то есть 1 или 2), а $i$ - номер стороны, где происходит $i$-е отражение (при этом здесь будем начинать с $i=0$ ). Заметим, что согласно этому определению, $\alpha_{0}^{1}$ и $\alpha_{0}^{2}$ совпадают с их введением в предыдущем абзаце (то есть обозначения корректны) и, кроме того, $x_{0}^{1}=x_{0}^{2}=x_{0}$ (см. координаты двух первых отражений на рис. 5). На этом рисунке угол многоугольника нарисован равным $\pi / 2$ вовсе не потому, что мы рассматриваем биллиард в форме правильного 4-угольника (квадрата), а просто потому, что так проще выполнить рисунок. Следующие точки отражения не нарисованы, более того, можно даже доказать, что никакая из двух траекторий на рис. 5 не может рассматриваться как начало бесконечной траектории, соответствующей $\omega$-слову $\gamma_{4}$. Однако, несмотря на это замечание, автор надеется, что рис. 5 все же понятен для восприятия.

Ниже мы приводим без доказательств леммы 1-5. Доказательства либо несложны (хотя и громоздки), либо могут быть получены с использованием фактов элементарной геометрии (и иллюстрируются рисунком 5).

Лемма 1. Справеливо неравенство $\alpha_{1}^{1}<\alpha_{1}^{2}$.

Лемма 2. Для $j=1,2$ справедливы равенства

$$
\alpha_{0}^{j}=\alpha_{2}^{j}=\alpha_{4}^{j}=\ldots=\alpha_{2 n}^{j}=\ldots
$$

$u$

$$
\alpha_{1}^{j}=\alpha_{3}^{j}=\alpha_{5}^{j}=\ldots=\alpha_{2 n+1}^{j}=\ldots
$$

Итак, согласно леммам 1 и 2 для каждого четного $i$ справедливо неравенство $\alpha_{i}^{1}>\alpha_{i}^{2}$. Аналогично $\alpha_{i}^{1}<\alpha_{i}^{2}$ для каждого нечетного $i$. 
Лемма 3. Пусть для некоторого $i \geqslant 0$ выполнены условия $x_{i}^{1} \leqslant x_{i}^{2} u \alpha_{i}^{1}>\alpha_{i}^{2}$. Тогда $x_{i+1}^{1}>x_{i+1}^{2}$, более того,

$$
\Delta_{i}=\left|x_{i+1}^{1}-x_{i+1}^{2}\right|-\left|x_{i}^{1}-x_{i}^{2}\right|>0 .
$$

Лемма 4. Пусть для некоторого $i \geqslant 0$ выполнены условия $x_{i}^{1} \geqslant x_{i}^{2} u \alpha_{i}^{1}<\alpha_{i}^{2}$. Тогда $x_{i+1}^{1}<x_{i+1}^{2}$, и более того, число $\Delta_{i}$, определяемое согласно (2), положительно.

Лемма 5. Справедливы неравенства

$$
\Delta_{0}<\Delta_{1}<\Delta_{2}<\ldots
$$

Итак, согласно леммам 1-5, не существует двух бесконечных последовательностей $\left(x_{i}^{1}\right)$ и $\left(x_{i}^{2}\right)$ (поскольку длина стороны рассматриваемого многоугольника конечна). Поэтому наше предположение, что оба выходные $\omega$-слова равны $\gamma_{n k}$, неверно. Полученное противоречие доказывает предложение 3.

Теорема 4. Для различных $k$ фигуры отражений, соответствующие слову $u_{n k} c$ подчеркнутой последней буквой, различньл.

Доказательство. Рассмотрим фигуру отражений для слова 이. Заметим, что мы уже рисовали такую фигуру для $n=6$ в разделе 4 . Обозначим эту фигуру $G$.

Обозначим буквы определенного выше $\omega$-слова $\gamma_{n}$ следующим образом:

$$
\gamma_{n}=a_{1} a_{2} \ldots
$$

(например, всегда, для каждого возможного $n, a_{1}=0, a_{2}=1$ и $a_{3}=2$ ).

Теперь рассмотрим фигуры отражений для

$$
a_{1} a_{2} \ldots a_{k-1} \underline{a_{k}}
$$

для каждого $k \geqslant 1$, обозначим их $F_{k}$. Рассмотрим также фигуры отражений для

$$
a_{1} a_{2} \ldots a_{k-1} \underline{a_{k}} a_{k+1}
$$

также для каждого $k \geqslant 1$, обозначим их $G_{k}$. По определению фигур отражений,

$$
G_{k}=F_{k} \cap G
$$

(этот факт может быть проиллюстрирован, например, вторым примером раздела 4).

Согласно (3), получаем цепочку включений

$$
G_{1} \supseteq G_{2} \supseteq G_{3} \supseteq \ldots \supseteq G_{i} \supseteq G_{i+1} \supseteq \ldots
$$

Заметим, что согласно предложению 1 каждая из фигур $G_{i}$ содержит по крайней мере 2 различные точки, имеющие одинаковые первые координаты.

Предположим, что существует некоторое $i$ такое, что $G_{i}=G_{i+1}$. Заметим, что согласно этому предположению, мы имели бы равенства

$$
G_{i}=G_{i+1}=G_{i+2}=\ldots
$$

что означало бы, что последовательность (4) стабилизируется. Поэтому фигура отражений рассматриваемого $\omega$-слова $\gamma_{n}$ имела бы по крайней мере 2 точки с одинаковыми 
первыми координатами. Последняя фигура отражений определяется обычным для выходов способом, а именно, как множество точек (прямоугольника $[0,1] \times[-\pi / 2, \pi / 2])$, соответствующих отражению, после которого получаемое нами согласно разделу $3 \omega$-слово совпадает с рассматриваемым. В статье мы не будем рассматривать такие фигуры, мы лишь покажем, что они не могут иметь 2 или более точек, имеющих одну и ту же первую координату.

Наличие двух точек с одинаковыми первыми координатами противоречит предложениям 2 и 3.

Таким образом, последовательность (4) не стабилизируется, то есть

$$
G_{1} \supset G_{2} \supset G_{3} \supset \ldots \supset G_{i} \supset G_{i+1} \supset \ldots
$$

Последний факт доказывает теорему.

Мы доказали, что существует бесконечное множество фигур отражений. Более того, согласно предложению 1 каждая из таких фигур содержит по крайней мере 2 различные точки, имеющие одну и ту же первую координату.

\section{6. Различные входные фигуры определяют различные выходные $\omega$-языки}

В этом разделе мы докажем факт, касающийся простой связи между входными фигурами отражений и выходными $\omega$-языками соответствующих им состояний рассмотренных $\omega$-автоматов.

Теорема 5. Различные входные фигуры отражений определяют различные выходные $\omega-я з ы к и$.

Как и ранее (для предложений 2 и 3), мы не будем рассматривать общий случай, то есть саму теорему 5; мы докажем только частный случай этой теоремы, необходимый для дальнейшего изложения.

Теорема 6. Различные входные фигуры отражений, соответствующие различным префиксам $\omega$-слова

$$
\gamma_{n}=012 \ldots m 012 \ldots m \ldots,
$$

определяют различные выходные $\omega$-языки.

Данная теорема является следствием предложения 3.

Итак, ни для какого $n \geqslant 3$ мы не можем определить $\omega$-язык правильного $n$-угольника с помощью $\omega$-КА с конечным числом состояний, поскольку каждое подмножество эквивалентных состояний $\omega$-КА состоит из единственного элемента.

\section{7. Языки обструкций правильных многоугольников не являются регулярными}

Формулировка следующей теоремы, основной теоремы статьи, совпадает с названием раздела; фактически, она просто является следствием фактов, сформулированных во всех предыдущих предложениях и теоремах. 
Теорема 7. Языки обструкций правильных многоугольников не являются регулярными.

Доказательство. Пусть для некоторого $n \geqslant 3$ соответствующий язык обструкций регулярен. В этом случае соответствующий $\omega$-КА (имеющий бесконечное множество состояний) также должен иметь регулярный язык обструкций.

Без ограничения общности мы можем зафиксировать сторону первого отражения (то есть первую букву рассматриваемого $\omega$-слова); в этом случае соответствующий $\omega$-автомат, как следует из изложенного в разделе 4.3, будет детерминированным. Согласно теореме 3 существует разбиение множества его состояний на конечное число подмножеств эквивалентных состояний (то есть состояний, имеющих одинаковые выходные $\omega$-языки). А согласно изложенному в разделах 5 и 6 мы не можем определить $\omega$-язык правильного многоугольника с помощью $\omega$-автомата с конечным числом состояний.

\section{8. Заключение}

Сформулируем несколько групп задач для дальнейшего решения. Мы уже доказали, что язык обструкций для биллиардных $\omega$-языков (в случае, когда биллиард имеет форму правильного многоугольника) не является регулярным. Однако неясно, как наиболее просто описать такие языки обструкций; например, еще надо ответить на вопрос, может ли какой-либо из таких языков быть контекстно-свободным.

Следующая проблема - исследовать, совпадает ли язык обструкций для $\omega$-языка квадрата с одним из языков, рассмотренных в [20] (подробное определение языка см. в [20]).

Существует еще группа задач, связанных с биллиардами, имеющими форму $n$-угольника, вообще говоря, не являющегося правильным. Для каждого из таких многоугольников мы можем определить его язык обструкций таким же способом, как выше это было сделано для правильных многоугольников. После этого для каждого $n \geqslant 3$ мы получаем две разные задачи, задачи описания языков, состоящих из следующих слов:

- слово является обструкцией для каждого $n$-угольника (например, для всех $n \geqslant 3$ таким словом является 00);

- слово является обструкцией для некоторого $n$-угольника (например, существует треугольник с обструкцией 01210, которая, как несложно убедиться, не является обструкцией для правильного треугольника, с помощью фактов элементарной геометрии можно доказать, что в качестве примера можно рассматривать равнобедренный треугольник с углами $10^{\circ}, 85^{\circ}$ и $85^{\circ}$, причем 2 - метка основания.

Заметим, что такие языки в обоих случаях не совпадают с языками обструкщий какоголибо конкретного многоугольника, однако этот факт выходит за рамки настоящей статьи.

Как уже было отмечено, мы не знаем, является ли язык обструкций некоторого заданного многоугольника контекстно-свободным. Однако несложно доказать, что любой из таких языков может быть определен с помощью некоторой машины Тьюринга, и следовательно, принадлежит иерархии Хомского [17]. Такая машина Тьюринга должна проверять непустоту каждой из фигур отражений, соответствующих каждому подслову рассматриваемого слова. Для этого она сначала вычисляет уравнения линий, ограничивающих эти фигуры отражений.

Кратко опишем еще одну группу задач для дальнейшего рассмотрения. Аналогично [6], мы можем рассматривать траектории, являющиеся бесконечными в обе стороны $(2 \omega$-слова), и формулировать для этих траекторий и соответствующих $2 \omega$-автоматов практически те же задачи, которые рассматривались в настоящей статье для $\omega$-слов. 


\section{Список литературы}

1. Eilenberg S., Automata, languages and machines. Academic Press, New York, 1974.

2. Саломаа А., Жемчужины теории формальных языков. Мир, Москва, 1986.

3. Thomas W., Automata on infinite objects. In: Formal models and semantics. Handbook of theoretical computer science (van Leeuwen, J., Ed.), v. B. Elsevier, Amsterdam, 1990, pp. 133-191.

4. Staiger L., $\omega$-Languages. In: Handbook of formal languages, v. 3. Springer, Berlin, 1997, pp. 339387.

5. Брауэр В., Введение в теорию конечных автоматов. Радио и связь, Москва, 1987.

6. Melnikov B., $2 \omega$-finite automata and sets of obstructions of their languages. Korean J. Comput. Applied Math. (1999) 6, №3, 565-574.

7. Bergman G. M., The diamond lemma for ring theory. Adv. Math. (1978) 29, 178-218.

8. Уфнаровский В., Комбинаторные и асимптотические методы в алгебре. Итоги науки и техники, Сер. Современные проблемы математики, фундаментальные направления, т. 57. ВИНИТИ, Москва, 1990, с. 5-177.

9. Belov A., Borisenko V., Latyshev V., Monomial algebras. J. Math. Sci. (1997) 87, №3, 3463-3575.

10. Трахтенброт Б. А., Бардзинь Я. М., Конечные автоматы: поведение и синтез. Наука, Москва, 1970.

11. Staiger L., Finite-state $\omega$-languages. J. CSS (1983) 27, 434-448.

12. Beal M. P., Minimal forbidden words and symbolic dynamics. In: Proc. STACS'96, pp. 555-566.

13. Maler O., Staiger L., On syntactic congruences for $\omega$-languages. TCS (1997) 183, 93-112.

14. Mignosi F., Forbidden factors in finite and infinite words. In: Jewels are Forever. Springer, Berlin, 1999, pp. 339-350.

15. Perrin D., Finite automata. In: Formal models and semantics. Handbook of theoretical computer science (van Leeuwen, J., Ed.), v. B. Elsevier, Amsterdam, 1990, pp. 1-57.

16. Cohen R. S., Gold Y., Theory of $\omega$-languages. I. Characterizations of $\omega$-context-free languages. $J$. Comp. and System Sci. (1977) 15, 169-184.

17. Ахо А., Ульман Дж., Теория синтаксического анализа, перевода и компилячии, т. 1. Мир, Москва, 1978.

18. Melnikov B., Vakhitova A., Some more on the finite automata. Korean J. Comput. Applied Math. (1998) 5, №3, 495-506.

19. Melnikov B., Melnikova A., Some properties of the basis finite automaton. Korean J. Comput. Applied Math. (2002) 9, № 1, 131-150.

20. Bridson M., Gilman R., Formal language theory and the geometry of 3-manifolds. Comment. Math. Helv. (1996) 71, №4, 525-555. 